\title{
Spontaneous Plugging of the Horizontal Semicircular Canal With Reversible Canal Dysfunction and Recovery of Vestibular Evoked Myogenic Potentials
}

\author{
$* \dagger+$ Leonel Luis, $\$$ João Costa, §Fernando Vaz Garcia, ||Josep Valls-Solé, \\ \Thomas Brandt, and $\mathbf{q}$ Erich Schneider \\ *Health Sciences Institute, Portuguese Catholic University; $\dagger$ Clinical Physiology Translational Unit, $\ddagger$ Institute \\ of Molecular Medicine, Faculty of Medicine, University of Lisbon; and \$EQUI-Clínica da Vertigem e \\ Desequilibrio, Lisbon, Portugal; \|EMG and Motor Control Unit, Neurology \\ Department, Hospital Clinic, Universitat de Barcelona, IDIBAPS, Spain; IInstitute for Clinical Neurosciences \\ and German Vertigo/Dizziness Center, IFB LMU, Ludwig-Maximilians University, Munich; \\ and \#Lausitz University of Applied Sciences, Senftenberg, Germany
}

\begin{abstract}
Objective: To evaluate the clinical pathophysiology of oculomotor changes in a patient presenting with a spontaneous semicircular horizontal canal plug.

Patient: A 42-year-old man with acute spontaneous vertigo with spinning and persistent left-horizontal nystagmus, intensity but not direction dependent on head orientation with respect to gravity, indicating a benign paroxysmal positional vertigo due to otoconia causing a plug in the horizontal semicircular canal. Intervention: Electrophysiological and video-oculographic testing; vestibular rehabilitation.

Main Outcome Measures: Cervical and ocular vestibular evoked myogenic potentials (VEMPs); video head impulse testing.

Results: The video head-impulse test revealed an eye velocity cutoff at $80 \%$ s in the time interval from 40 to $90 \mathrm{~ms}$ after initiation of head impulses to the right. This normalized within 2 days after liberatory maneuvers, documenting for the first time a reversible deficiency of the cupular-endolymph highfrequency system dynamics. Cervical and ocular vestibular
\end{abstract}

myogenic potentials were absent during stimulation of the affected side before the liberatory maneuvers but normalized within 30 to 80 days.

Conclusion: This case is special in 4 respects: 1) nystagmus intensity, but not direction, was dependent on head orientation with respect to gravity, indicating a horizontal canal plug; 2) VEMPs were asymmetrical before liberatory maneuvers; 3) VEMPs recovered after Day 30; and 4) video head-impulse test asymmetry recovered. These observations challenge the common belief that VEMPs are evoked by otolith stimulation only. Instead, the assumption of a reversible canal dysfunction by a plug offers a more plausible explanation for all effects. Key Words: Benign paroxysmal positional vertigo-Reflexvestibulo-ocular-Vestibular function tests.

Otol Neurotol 34:743-747, 2013.
Benign paroxysmal positional vertigo (BPPV) is caused by otoconial debris that dislodge from the utricular macula and precipitate into the semicircular canals (SCCs),

Address correspondence and reprint requests to Leonel Luis, M.D., Health Sciences Institute, Portuguese Catholic University, Edifício da Biblioteca João Paulo II - $5^{\circ}$ piso, Palma de Cima, 1649-023 Lisbon, Portugal; E-mail: leonelluis@me.com

This study was supported in part by the German Federal Ministry of Education and Research (grant no. 01 EO 0901).

The authors disclose no conflicts of interest.

Supplemental digital content is available in the text. thereby disturbing the endolymph flow during head movement. The debris can also fall into the ampulla, making the cupula sensitive to gravity (1). There are 2 types of BPPV with horizontal nystagmus (hBPPV). The clinically more frequent type with geotropic nystagmus is supposedly caused by canalolithiasis of the long SCC arm, whereas the less frequent hBPPV, characterized by apogeotropic horizontal nystagmus during the PagniniMcClure maneuver, is thought to be caused either by canalolithiasis of the SCC short arm or by cupulolithiasis. Both types can convert into the other (2). Patients usually have a history of transient or persistent positional vertigo, 
but can present with spontaneous vertigo and spontaneous nystagmus $(\mathrm{SN})(3,4)$ due to cupulolithiasis or spontaneous canal plugging.

The main goal of the vestibulo-ocular reflex (VOR) is to maintain clear vision by retinal image stabilization of the visual surroundings, particularly important during rapid head movements. The VOR function can be quantitatively assessed with the video head-impulse test (vHIT) $(5,6)$, which records and analyzes the velocity trajectory of eye movements along with and during high-velocity head impulses. The otolithic-ocular reflex is also an integral part of the VOR. This is thought to be evaluated by ocular vestibular evoked myogenic potentials (oVEMPs) in the same way that the vestibulocollic reflex can be accessed through cervical vestibular evoked myogenic potentials (cVEMPs) (7).

We report on a case that challenges the common belief that VEMPs are evoked by otolith stimulation only and document for the first time a high-frequency VOR hypofunction during BPPV.

\section{CASE REPORT}

A 42-year-old man was admitted to the emergency room with acute spontaneous and spinning vertigo with nausea and vomiting. He had no cochlear symptoms, history of trauma, or previous complaints of vertigo, dizziness, vestibular, visual, or postural symptoms. Examination revealed a Grade III left horizontal SN.

During positional testing, the intensity, but not the direction, of the nystagmus changed (stopping with the head pitched down approximately $30^{\circ}$ and decreasing during leaning, in the left Pagnini-McClure and left Dix-Hallpike maneuvers). During the Pagnini-McClure maneuver, nystagmus was apogeotropic on the right and geotropic (and weaker) on the left.

The neurological examination was otherwise unremarkable, as was the clinical head-impulse test (HIT). Result of the brain magnetic resonance imaging was normal. Because assessment of the pathophysiology and identification of the affected side were not possible clinically, vHIT and VEMPs were performed.

A video-oculography device $\left(\right.$ EyeSeeCam $\left.^{\circledR}\right)$ was used to perform the vHIT. Three trials ( 20 head impulses each) were passively and randomly applied toward either side in the plane of the horizontal SCC to check for reproducibility. The VOR gain was significantly reduced in head impulses to the right but was normal to the left (head velocities from 200 to $300 \%$ s). From 40 to $90 \mathrm{~ms}$ after head movement initiation, the eye velocity saturated and remained constant at $80 \%$, yielding a median gain of 0.29 at $60 \mathrm{~ms}$ (Fig. 1). Left overt compensatory saccades
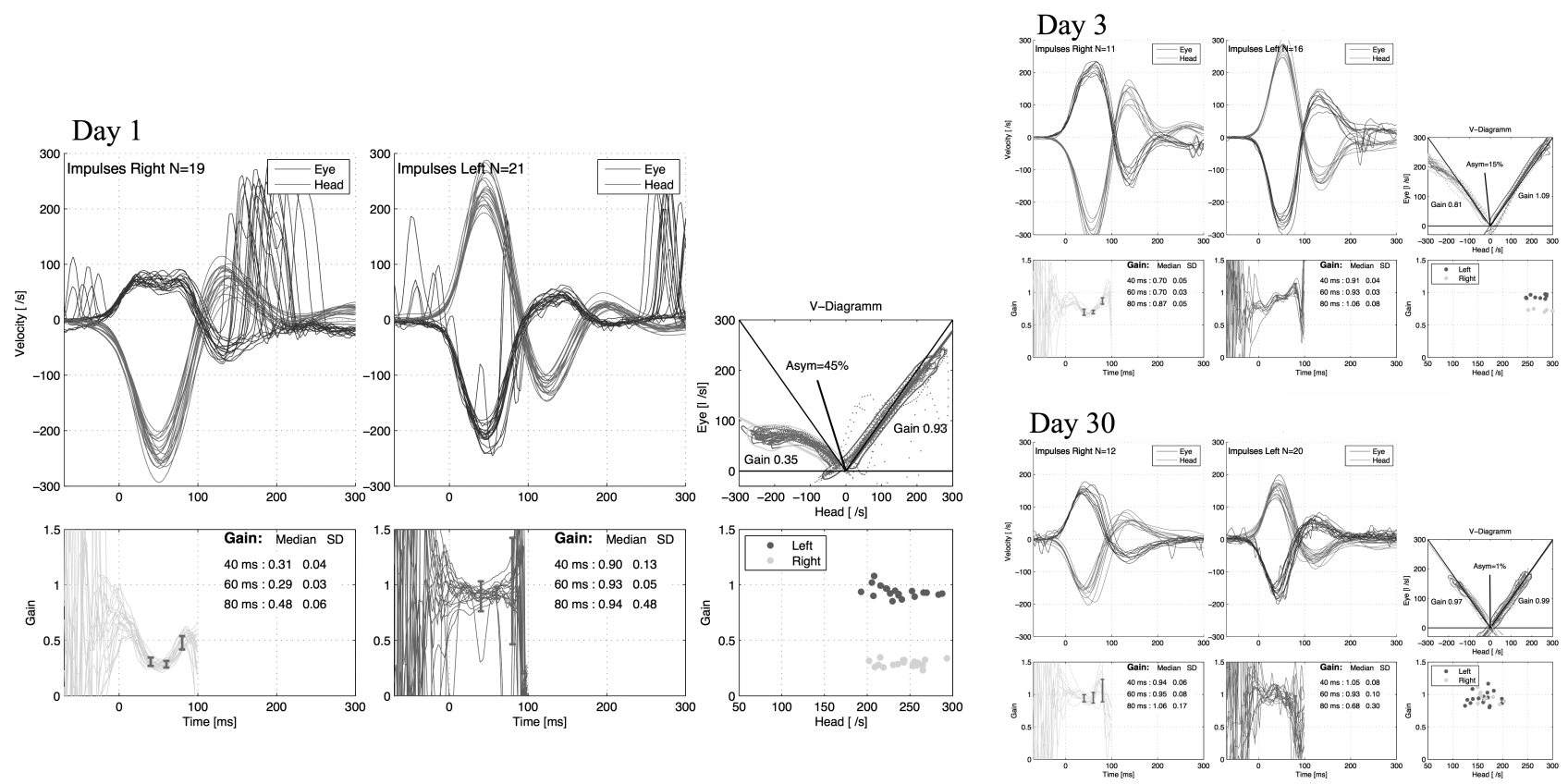

FIG. 1. Video head-impulse test (VHIT) recordings. In each trial and for all valid head impulses, the 2 left upper plots show the velocity trajectories ( $\%$ ) of the eye (dark grey lines) and head (light grey lines) and the right upper plot the regressions of eye and head velocity during right and left impulses; the 2 left lower plots show the time course (ms) of the VOR gain (eye velocity/head velocity) and the right lower plot the VOR gain values at $60 \mathrm{~ms}$. vHIT on Day 1. During right HIT, note the saturated profile of the eye velocity curve and the low VOR gain $(0.31,0.29$, and 0.48 at 40,60 , and $80 \mathrm{~ms}$, respectively), as well as the corrective saccades triggered from 120 to $260 \mathrm{~ms}$. Note also the saccades during left HIT, randomly present in the first $100 \mathrm{~ms}$ but also later locked at around 300 ms (corresponding to the fast phase of the spontaneous nystagmus). For rightward head impulses, the eye velocity saturated and remained constant at $80 \%$ with head velocities above $150 \%$. This saturation can also be observed in the regression diagram and in the eye and head velocity trajectories. vHIT on Day 3. The eye velocity curve regained its normal trajectory, although the VOR gain still shows a slight asymmetry. vHIT on Day $\mathbf{3 0}$. The eye velocity curve has a completely normal trajectory, and the VOR gain is symmetrical. No saccades were triggered. 
were triggered during right impulses (120-260 ms latency). Anticompensatory quick phases were triggered by left impulses and locked at 260 to $320 \mathrm{~ms}$. Both cVEMPs and oVEMPs were absent after right-side stimulation (Fig. 2).

With only the results of the clinical examination, this patient would most probably have been diagnosed to have right vestibular neuritis and treated accordingly. It was, in particular, the remarkable saturation of eye velocities during head impulses to the right that led us to hypothesize an otoconia plug in the right horizontal canal. To the best of our knowledge, such a peculiar eye velocity trajectory has never been described in the literature before, for example, as a characteristic for vestibular neuritis or any other known condition. On the basis of this high-frequency VOR deficit and the gravity-dependent $\mathrm{SN}$, as well as the observation that the Pagnini-McClure maneuver did not cause geotropic nystagmus on both sides, the alternative hypothesis of a right horizontal canalolithiasis was also rejected.

The examining first author therefore decided to perform a liberatory maneuver by positioning the patient from sitting to lying on the right side and then turning his head quickly down $45^{\circ}$. In contrast to a Gufoni maneuver, which would have generated an endolymph ampullofugal movement, this maneuver used the increased inertia of the hypothesized otoconial canal plug. When the patient returned to a sitting position, a right-beating horizontal nystagmus appeared (see Video, Supplemental Digital Content 1, http://links.lww.com/MAO/A144). A subsequent Pagnini-McClure maneuver test indicated that the debris had been mobilized in the canal because the nystagmus was now geotropic on both right and left. Then a $360^{\circ}$ body rotation around the longitudinal axis to the left was performed (8). This evoked a left-beating nystagmus during the first $180^{\circ}$ to $270^{\circ}$ rotation, suggesting that the debris moved along the long arm of the right horizontal SCC back into the utricular cavity. The patient was free of symptoms and $\mathrm{SN}$ when released from the emergency unit.

Forty-eight hours later, he was still symptom-free and clinical examination was unremarkable. The eye velocity profile during right vHIT was now normally shaped, with no cutoff, but there was still a slightly lower VOR gain on the right than on the left side $(0.81$ versus 1.09). Compensatory saccades were no longer triggered. Surprisingly, cVEMPs and oVEMPs were still absent, although the patient was now symptom-free.

Thirty days later, clinical examination was completely normal. The VHIT results were normal without VOR
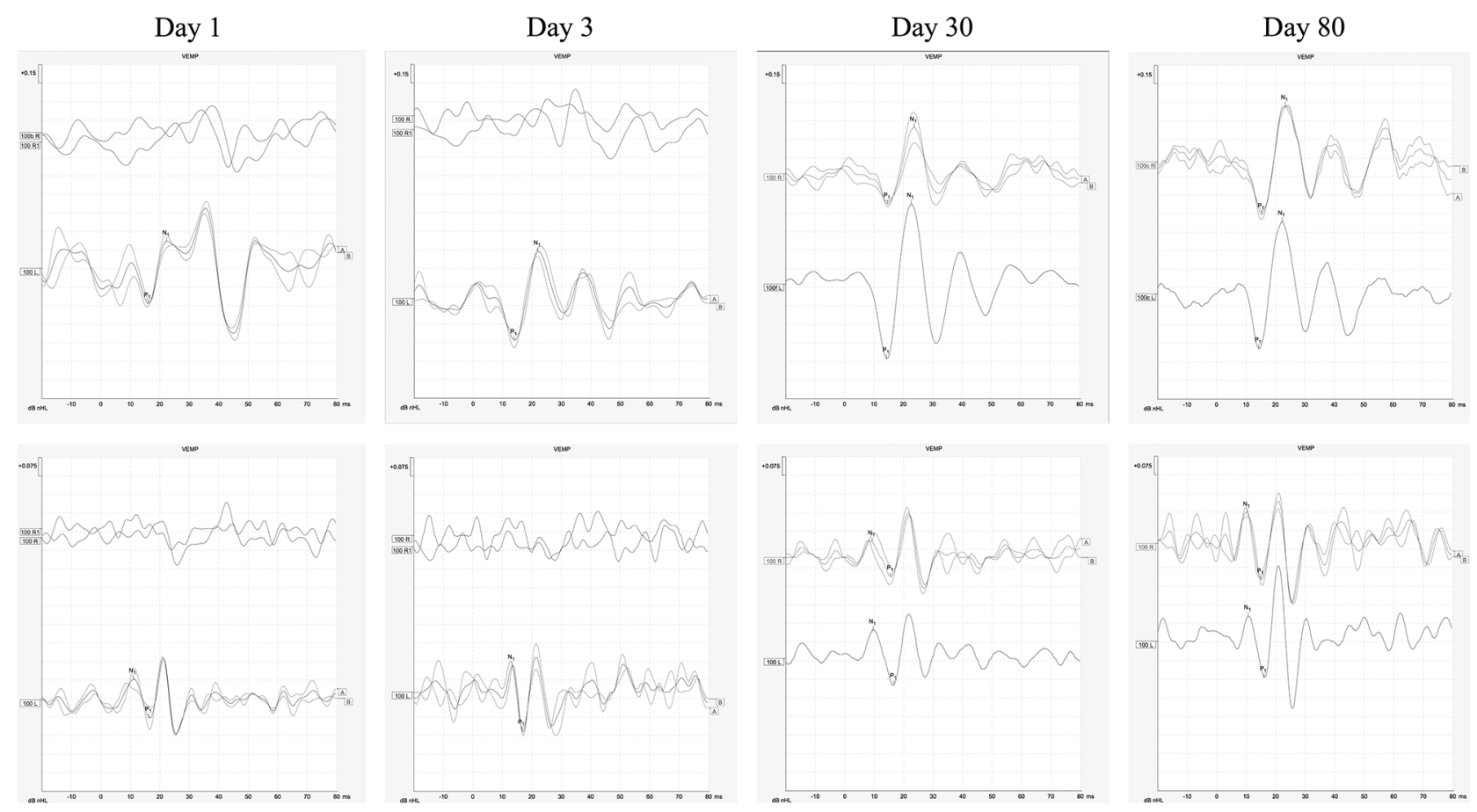

FIG. 2. Cervical (cVEMP; upper plots) and ocular (oVEMP; bottom plots) vestibular evoked myogenic potential recordings on Days 1,3 , 30, and 80. An Eclipse ${ }^{\circledR}$ vestibular EP from Interacoustics ${ }^{\circledR}$ was used for recordings. The vestibulocollic and vestibulo-ocular reflexes were evoked by rarefaction and a $500-\mathrm{Hz}$ tone burst (stimulation rate $=5.1 \mathrm{~Hz}, 100 \mathrm{~dB} \mathrm{nHL}$ ) delivered unilaterally to the homolateral (cVEMPs) and contralateral (oVEMPs) ear by a pair of insert earphones. A total of 200 and 500 recordings, respectively and separately, were averaged. Scaling of cVEMP and oVEMP amplitudes was applied according to muscle tonus level just before and immediately after each stimulus during the entire test. " $\mathrm{A}$ " curve holding all rarefaction sweeps and "B" curve holding all condensation sweeps were recorded independently and are shown for reproducibility. Day 1 . Note that right (R) cVEMPs and oVEMPs are absent and that left (L) cVEMPs and oVEMPs are normal. Day 3. Right (R) cVEMP and oVEMP are still absent and left (L) cVEMP and oVEMP are normal. Day 30. Right cVEMPs have normal morphology and latency. The interaural amplitude $(I A)$ difference with scaled curves $(I A=0.34)$ is already below the upper normal limit $(I A<0.35)$. The oVEMP responses are symmetrical $(I A=0.21)$. Day 80 . Both cVEMPs and oVEMPs are symmetrical with IA of 0.08 and 0.05 , respectively. 
gain asymmetry between the sides. Both cVEMPs and oVEMPs had normal bilateral trajectory and latency values, although a slight interaural amplitude difference was still present. This completely recovered by Day 80 .

Eight months after this first event the patient was diagnosed to have a right geotropic hBPPV. A mild asymmetry between sides was again recorded with vHIT and cVEMP, later recovering by Day 30 (further details of the second episode are provided as Supplementary Material: See Text, Supplemental Digital Content 3, http://links.lww.com/ MAO/A146 and Figure, Supplemental Digital Content 4, http://links.lww.com/MAO/A147).

\section{DISCUSSION}

This case is special in 4 respects: 1) the properties of the SN, which depended on head orientation with respect to gravity, indicating that a plug of otoconia debris blocked the right SCC; 2) the reversibility of a high horizontal VOR function asymmetry documented in the vHIT, early after liberatory maneuvers; 3) the high asymmetry of both cVEMPs and oVEMPs before and 2 days after the liberatory maneuvers; and 4) VEMP recovery 30 days after treatment.

\section{Clinical Course of Horizontal Semicircular Canal Plugging}

The vHIT was used for the first time to document a high-frequency VOR hypofunction during BPPV that improved within 2 days after treatment and returned to normal within 30 days. Of particular interest was a remarkable eye velocity trajectory that saturated at approximately $80 \%$ s as if the cupula was kept at a constant deflection, regardless if head velocity increases up to $300 \%$ s. Reversible low-frequency VOR hypofunction has been previously reported in hBPPV canalolithiasis using calorics (9).

The most plausible explanation for both the saturation of the eye velocity response and the nystagmus pattern is a canal plug by otoconia. It is thought that such a plug causes a negative cupular pressure and blocks endolymph flow (see Picture, Supplemental Digital Content 2, http://links.lww.com/MAO/A145) (10). The leftbeating $\mathrm{SN}$ in the upright position was probably due to the $30^{\circ}$ inclination of the horizontal SCC (3) because a null point was identified with the head pitched down approximately $30^{\circ}$. Because its direction was not modified by the different head positions, this nystagmus was not a pseudo-SN (3). Such would be expected if the otoconia debris were to move to the most gravitydependent part of the canal.

The asymmetrically saturated eye velocity trajectory cannot methodologically be ascribed to goggle slippage. Goggle slippage causes eye-to-head velocity asynchrony in both head movement directions rather than systematic eye velocity saturation in just one direction. In the other direction, the eye and head velocity trajectories were mirror-symmetrical. Had goggle slippage caused the observed velocity saturation, the liberatory maneuver would not have restored mirror symmetry on the affected side. This can only be the result of a reversible biomechanical modification of the cupular-endolymph system dynamics by the canal plug.

Left saccades were also identified during left vHIT, probably corresponding to the fast phase of the leftbeating nystagmus. It is possible that the head impulses generated a short, but high-amplitude increase in the level of persisting vestibular imbalance, which in turn triggered quick phases locked at 200 to $300 \mathrm{~ms}$ after impulse onset. A mild dynamic VOR gain asymmetry persisted on Day 3, probably due to continuing changes in biomechanical SCC properties.

\section{Horizontal Semicircular Canal Plugging Affects cVEMPs and oVEMPs}

Both cVEMPs and oVEMPs were absent on the right side at presentation and after the liberatory maneuver. This is not the first report on BPPV affecting cVEMPs. Several studies have reported that $25 \%$ to $35 \%$ of BPPV patients have abnormal cVEMPs $(11,12)$, but most only evaluated posterior BPPV and no oVEMP data were published.

Interestingly, the symptoms and clinical examination did not correlate with the VEMP examination: The patient was symptom-free after the liberatory maneuver. This is surprising because the current understanding is that VEMPs test otolith function. On this basis, the positive VEMP result would suggest otolith dysfunction with accompanying symptoms, which, however, were not observed.

Later, VEMPs progressively normalized through Day 30 to Day 80 . An oVEMP recovery pattern has been reported 82 days after presentation in a vestibular neuritis patient in whom it is assumed that the lesion was confined to the nerve (13). Our case is different in that the absence of SN and vHIT normalization just after the liberatory maneuver excludes vestibular nerve involvement.

Our patient therefore had a mechanical canalar malfunction with normal vestibular nerve function. The absence of SN and the vHIT normalization after the liberatory maneuver do not suggest any other vestibular disturbances. The recovery from symptoms immediately after the liberatory maneuver raises the question of whether there was an associated otolith dysfunction at all. Such a dysfunction would necessarily have caused observable symptoms. We therefore hypothesize that SCC may contribute to VEMP responses.

The alternative explanation that the reported VEMP results are caused by an insult and early recovery of both otoliths is unlikely. It is difficult to conceive that receptor damage could recover as quickly and as completely as oVEMPs normalized. With such a receptor damage, ipsilateral cVEMPs and oVEMPs remained absent for at least 6 months in a patient with an acute peripheral cochleovestibular loss (14). The cVEMP and oVEMP recovery pattern reported here cannot be explained by the view that dynamic function of both saccule and utricle can simultaneously recover in less than 30 days $(13,14)$. 
No such simultaneous recovery process is known in the literature.

The interpretation that VEMPs mainly test the otoliths relies on animal studies in which the afferent units were classified as otolithic on the basis of their responsiveness to static head tilts $(15,16)$. This way, however, SCC units probably also entered the analysis because surgery exposed the animal labyrinth to a temperature gradient that made the SCC units gravity-dependent (17). A recent study that took account of this effect provided neuroanatomic and neurophysiological evidence of a possible contribution from both the canals and the otoliths to VEMP responses (17). Canal activation has also been proposed to be responsible for enhanced oVEMPs in superior SCC dehiscence $(18,19)$. Several studies found ipsilateral cVEMP threshold lowering (20) and oVEMP hypersensitivity (21) and cVEMP and oVEMP threshold normalization after superior canal plugging (22). Although threshold lowering and hypersensitivity of VEMP responses in the presence of canal dehiscence does not necessarily imply canal activation, it is interesting to note that the eye movements elicited in these patients occur in the plane of the affected canal. The differentially delayed normalization of both vHIT and VEMP responses after the liberatory maneuvers remains to be explained.

In conclusion, the observations presented here challenge the common belief that VEMPs are evoked by otolith stimulation only. In this case, the assumption of a reversible canal dysfunction by a plug offers a more plausible hypothetical explanation for all observations. In the future, the gain saturation observed with the vHIT might serve as a characteristic sign for a canal plug.

Acknowledgments: The authors thank Judy Benson for critically reading the article and Thierry Luis for drawing the illustrations.

\section{REFERENCES}

1. Brandt $T$, Steddin S. Current view of the mechanism of benign paroxysmal positioning vertigo: cupulolithiasis or canalolithiasis? $J$ Vestib Res 1993;3:373-82.

2. Steddin S, Brandt T. Horizontal canal benign paroxysmal positioning vertigo (h-BPPV): transition of canalolithiasis to cupulolithiasis. Ann Neurol 1996;40:918-22.

3. Asprella Libonati G. Pseudo-spontaneous nystagmus: a new sign to diagnose the affected side in lateral semicircular canal benign paroxysmal positional vertigo. Acta Otorhinolaryngol Ital 2008; 28:73-8.
4. Brevern von M, Clarke AH, Lempert T. Continuous vertigo and spontaneous nystagmus due to canalolithiasis of the horizontal canal. Neurology 2001;56:684-6.

5. MacDougall HG, Weber KP, McGarvie LA, Halmagyi GM, Curthoys IS. The video head impulse test: diagnostic accuracy in peripheral vestibulopathy. Neurology 2009;73:1134-41.

6. Bartl K, Lehnen N, Kohlbecher S, Schneider E. Head impulse testing using video-oculography. Ann N Y Acad Sci 2009;1164: 331-3.

7. Rosengren SM, Welgampola MS, Colebatch JG. Vestibular evoked myogenic potentials: past, present and future. Clin Neurophysiol 2010;121:636-51.

8. Lempert T. Horizontal benign positional vertigo. Neurology 1994; 44:2213-4.

9. Strupp M, Brandt T, Steddin S. Horizontal canal benign paroxysmal positioning vertigo: reversible ipsilateral caloric hypoexcitability caused by canalolithiasis? Neurology 1995;45:2072-6.

10. Sadeghi SG, Goldberg JM, Minor LB, Cullen KE. Effects of canal plugging on the vestibuloocular reflex and vestibular nerve discharge during passive and active head rotations. $J$ Neurophysiol 2009;102:2693-703.

11. Akkuzu G, Akkuzu B, Ozluoglu LN. Vestibular evoked myogenic potentials in benign paroxysmal positional vertigo and Ménière's disease. Eur Arch Otorhinolaryngol 2006;263:510-7.

12. Longo G, Onofri M, Pellicciari T, Quaranta N. Benign paroxysmal positional vertigo: is vestibular evoked myogenic potential testing useful? Acta Otolaryngol 2012;132:39-43.

13. Manzari L, Burgess AM, MacDougall HG, Curthoys IS. Objective verification of full recovery of dynamic vestibular function after superior vestibular neuritis. Laryngoscope 2011;121:2496-500.

14. Goto F, Ban Y, Tsutumi T. Acute audiovestibular deficit with complete ocular tilt reaction and absent VEMPs. Eur Arch Otorhinolaryngol 2011;268:1093-6.

15. Curthoys IS, Kim J, McPhedran SK, Camp AJ. Bone conducted vibration selectively activates irregular primary otolithic vestibular neurons in the guinea pig. Exp Brain Res 2006;175:256-67.

16. Curthoys IS, Vulovic V. Vestibular primary afferent responses to sound and vibration in the guinea pig. Exp Brain Res 2011;210: 347-52.

17. Zhu H, Tang X, Wei W, Mustain W, Xu Y, Zhou W. Click-evoked responses in vestibular afferents in rats. J Neurophysiol 2011; 106:754-63.

18. Welgampola MS, Migliaccio AA, Myrie OA, Minor LB, Carey JP. The human sound-evoked vestibulo-ocular reflex and its electromyographic correlate. Clin Neurophysiol 2009;120:158-66.

19. Aw ST, Welgampola MS, Bradshaw AP, Todd MJ, Magnussen JS, Halmagyi GM. Click-evoked vestibulo-ocular reflex distinguishes posterior from superior canal dehiscence. Neurology 2010;75: 933-5.

20. Niesten MEF, McKenna MJ, Herrmann BS, Grolman W, Lee DJ. Utility of cVEMPs in bilateral superior canal dehiscence syndrome. Laryngoscope 2013;123:226-32.

21. Zuniga MG, Janky KL, Nguyen KD, Welgampola MS, Carey JP. Ocular versus cervical VEMPs in the diagnosis of superior semicircular canal dehiscence syndrome. Otol Neurotol 2013;34:121-6.

22. Welgampola MS, Myrie OA, Minor LB, Carey JP. Vestibularevoked myogenic potential thresholds normalize on plugging superior canal dehiscence. Neurology 2008;70:464-72. 